Post-temporary ligation intra-operative mesenteric portovenography: comparison with computed tomographic angiography for investigation of portosystemic shunts

\title{
Andrew Parry
}

Willows Referral Service, Highlands Road, Shirley, Solihull, West Midlands B90 4NH

\section{Robert N White}

School of Veterinary Medicine and Science, University of Nottingham, Sutton Bonington Campus, 8 Leicestershire LE12 5RD

\section{SUMMARY}

Objectives: Comparison of pre-operative computed tomographic angiography and post-temporary full ligation intraoperative mesenteric portovenography for the documentation of the intrahepatic portal vasculature in patients with single extrahepatic portosystemic shunts.

Methods: Retrospective study of patients with extrahepatic portosystemic shunts that underwent preoperative computed tomographic angiography and intra-operative mesenteric portovenography after temporary full ligation of an identified shunt vessel. Studies were compared for appearance of the intrahepatic portal vasculature.

Results: Fourteen dogs and five cats were included in the study with various single congenital extrahepatic portosystemic shunts variations. With the exception of those shunts involving the right gastric vein, the identification of the intrahepatic arborisation was similar on both modalities. Subjectively, however, there was improved contrast enhancement, as well as slight enlargement of the intrahepatic portal vasculature, on portovenography compared to computed tomographic angiography.

Clinical significance: This paper shows that computed tomographic angiography cannot replace intraoperative mesenteric portovenography after temporary full ligation, which provides 
information regarding the development of intrahepatic portal vascularity. It is a practical and dynamic procedure providing results which are instantaneously available at the time of surgery. In addition, TFL-IOMP confirmed both that the shunting vessel had been recognised and only one vessel was present.

Keywords: Intraoperative mesenteric portovenography (IOMP), computed tomographic angiography (CTA), portosystemic shunt

\section{INTRODUCTION}

The use of computed tomography angiography (CTA) to describe both the anatomy of the normal portal vasculature and the anatomy of congenital portosystemic shunts in small animals is welldescribed (Frank et al. 2003, Zwingenberger \& Schwartz 2004, Zwingenberger et al. 2005, Echandi et al. 2007, Nelson \& Nelson 2011, White \& Parry 2013, 2015, 2016a, 2016b, Fukushima et al. 2014). For many years the use of intra-operative mesenteric portography (IOMP) was considered the "gold standard" for the diagnosis of portosystemic vascular abnormalities in the dog and cat (White et al. 2003). Recently, the morphology of the normal extrahepatic portal vein has been compared using both IOMP and CTA and it was concluded that CTA consistently showed more detail of the extrahepatic portal vein and its tributaries (Parry \& White 2015). In addition, in dogs and cats suffering from congenital extrahepatic portosystemic shunt (EHPSS), the pre-operative findings of CTA have been compared to the findings of shunt pre-ligation IOMP (Parry \& White 2017). This study concluded that pre-ligation IOMP was consistently outperformed by preoperative CTA in all cases except those patients suffering from an EHPSS involving the right gastric vein and that, as such, there could be little indication for performing pre-ligation IOMP in cases that had already undergone diagnostic preoperative CTA (Parry \& White 2017). 
IOMP can also be performed following the localisation and temporary full ligation of a congenital EHPSS (TFL-IOMP) and it is recognised that such studies provide different and clinically useful information when compared to IOMPs obtained prior to the surgical manipulation of the shunt. For example, TFL-IOMPs can confirm that the correct shunting vessel has been recognised and encircled correctly, and that further shunting vessels are not present (White et al. 2003, Lee et al. 2006, Lipscomb et al. 2009). In addition, TFL-IOMP has been shown to provide an indication of the presence and degree of development of the patient's intrahepatic portal vasculature; information used at the time of surgery to help the surgeon decide whether or how the shunt should be attenuated (White et al. 2003, Lee et al. 2006, Lipscomb et al. 2009). The information gained from a TFLIOMP has been shown to have a positive correlation with the prognosis of patients undergoing shunt attenuation surgery; the more developed the intrahepatic portal vasculature, the better the long-term prognosis following shunt closure surgery (Lee et al. 2006, Lipscomb et al. 2009).

The purpose of this study was to compare and contrast the imaging modalities of pre-operative CTA and TFL-IOMP in dogs and cats suffering from a congenital EHPSS, and to use this information to make recommendations for their use in the clinical management with congenital EHPSSs.

\section{METHODS}

This retrospective study reviewed dogs and cats seen by the authors between 2009 and 2016 for the investigation and management of a congenital EHPSSs. The inclusion criteria were that all cases must have a congenital EHPSS, have had a pre-operative CTA within four weeks prior to surgery and have undergone recorded IOMP after temporary full ligation of the shunting vessel (TFL-IOMP).

Data on breed, signalment (age, sex, neutering status), imaging investigation, type of portosystemic 
shunt and gross surgical findings were collected and reviewed.

CTA was performed under anaesthesia using a 16 slice multidetector unit (Brightspeed, General Electric Medical Systems, Milwaukee) as described previously (White and Parry 2016a, 2016b). Briefly, images were acquired using a $0.625 \mathrm{~mm}$ or $1.25 \mathrm{~mm}$ slice collimation, depending on the size of the animal, $120 \mathrm{kVp}$ and variable mAs. Patients were positioned in sternal recumbency and apnoea was induced prior to image acquisition to reduce respiratory motion. Scanned field of view (SFOV) and displayed field of view (DFOV) were selected according to the size of the animal. The collimator pitch was 0.938 . Pre- and post-intravenous contrast (600mg I/kg, Iohexol, Omnipaque, GE Healthcare, Norway) images were obtained using a standard algorithm (medium frequency reconstruction kernel) and a 512 x 512 matrix, and viewed using a window and level optimised for soft tissue (window 400HU, level 50HU). Contrast was injected at a speed of $2.0-3.0 \mathrm{ml} / \mathrm{s}$ (depending on the size of the animal and consequently the size of intravenous catheter placed) using a pressure injector (Medrad Stellant CT injection system, Bayer Healthcare Medical Care, Indianola). To optimise contrast enhancement, a transverse slice over the mid-abdomen was selected and repetitively examined whilst contrast injection was performed. At the onset of opacification of the portal vessels, a complete abdominal dual phase CTA examination was performed using proprietary bolus tracking software with an automated trigger threshold of $120 \mathrm{HU}$ to start the scan. The trigger region of interest was positioned over the portal vein at the level of the porta hepatis in all dogs and cats, in the central aspect of the vessel to allow for respiratory motion. A further tissue pool phase was then performed without using bolus tracking. Studies were assessed in their native format, using multiplanar reformatting (MPR) and maximum intensity projection (MIP). Volume rendered images were not utilized. All CTA studies were reviewed by both authors.

TFL-IOMP was carried out during surgery with the patients positioned in dorsal recumbency. A 
mobile image intensification unit (OEC Fluorostar 7900, General Electric Medical Systems, Milwaukee) was used to obtain ventrodorsal images of the cranial abdomen following the temporary, full ligation of the shunting vessel (White et al. 1996, White et al. 1998). A jejunal vein was cannulated with a catheter (20 or 22 gauge). The shunting vessel was located and encircled with a ligature of either 3-0 or 2-0 polypropylene (Prolene, Ethicon UK Ltd.) close to its communication with the systemic vein. The shunt was temporarily fully closed using the encircling ligature as a vascular snare and although the time of total occlusion was not measured specifically, in none of the cases was the total occlusion time more than 90 seconds. Although mesenteric (portal) venous pressures were not specifically recorded, they were assessed to ensure that they did not exceed $20 \mathrm{cmH}_{2} \mathrm{O}$ during the period of TFL-IOMP in any of the cases. A mask was applied to create a digital subtraction angiogram and a bolus of non-ionic iodinated contrast agent (iohexol, Omnipaque, GE Healthcare, Norway) was injected into the jejunal vein to produce the images. The total dose of iodine did not exceed $600 \mathrm{mg} \mathrm{I} / \mathrm{kg}$. The contrast was injected by hand using a 10 or $20 \mathrm{ml}$ syringe. Angiograms were recorded digitally and were reviewed retrospectively by both authors as video loops.

The CTA and TFL-IOMP images were evaluated using a method adapted from those described previously (Macdonald et al. 2002, Zwingenberger \& Schwarz 2004, Lee et al. 2006, Parry \& White 2015). Intrahepatic portal vein arborisation was assessed for the presence or absence of a portal vein entering the liver; principal right and left portal branches; branching of the principal portal branches; and primary, secondary and tertiary branching of the principal branches (Macdonald et al. 2002). Furthermore, a subjective assessment of the size and opacity (attenuation) of the intrahepatic vessels was made between the two modalities.

The TFL-IOMP and CTA data were reviewed in a random order using simple randomisation of the data. 


\section{RESULTS}

Fourteen dogs and 5 cats met the inclusion criteria. No patients were excluded due to image quality. Three dogs had a shunt emanating from the left gastric vein, of which 2 had a left gastrophrenic shunt and 1 had a left gastroazygos shunt (White \& Parry 2013). Six dogs had a shunt involving the right gastric vein, of which 3 dogs had a type Ai, 1 dog had a type Aii and 2 dogs had a type Aiii (no dogs had a type B shunt) (White \& Parry 2015). Three dogs had a splenocaval shunt (White \& Parry 2016a). Two dogs had a shunt involving the left colic vein, of which 1 dog had a shunt entering the caudal vena cava and 1 dog had a shunt entering the cranial rectal vein (White \& Parry 2016b). Of the 5 cats, 2 had a left gastrophrenic shunt, 1 cat had a left gastrocaval shunt, 1 cat had a splenocaval shunt, and 1 cat had a shunt involving the left colic vein (which inserted into the caudal vena cava). These extrahepatic findings on CTA were confirmed at surgery.

The age, breed and sex distribution of the patients with various different shunt types were consistent with previous studies. More shunts involving the right gastric vein were identified in this study than in previous studies (see table 1).

\section{CTA:}

In all cases, CTA documented the presence of a portal vein entering the liver. There was however variation in the appearance of intrahepatic arborisation according to shunt type. In all left gastrophrenic, left gastrocaval, left gastroazygos, and splenocaval shunts, as well as the three shunts involving the left colic vein, CTA documented the presence of the portal vein entering the liver, the principal right and left portal branches, the primary, secondary and tertiary branching of the principal branches and the opacification of the right and left lobes of the liver. In all patients with shunts involving the right gastric vein, CTA documented the presence of the portal vein entering 
the liver. However, of these 6 cases, the principal right and left portal branches were only identified in 5/6 dogs, and the primary, secondary and tertiary branching of the principal branches in 3/6 dogs.

\section{TFL-IOMP:}

In all cases, the presence of the portal vein entering the liver and the principal right and left portal branches were identified. The primary, secondary and tertiary branching of the principal branches was seen in all cases (see table 2).

Subjectively, the divisions of the intrahepatic portal branches appeared slightly larger and more intensely contrast enhancing on IOMP images compared to the CTA images. This subjective finding was consistent in all IOMP video loops compared to the corresponding CTA images (see figure 1).

No patients suffered any obvious or apparent intra-operative complications (for example, changes in anaesthetic monitoring physiological parameters) as a result of the temporary full occlusion of the shunt or the TFL-IOMP.

\section{DISCUSSION:}

With the exception of those shunts involving the right gastric vein, the identification of the intrahepatic arborisation on CTA and TFL-IOMP was similar, with all primary, secondary and tertiary portal divisions identified. Subjectively, however, there was improved contrast enhancement, as well as slight enlargement of the intrahepatic portal vasculature, on TFLIOMP when compared to CTA. This finding is intuitive. The non-selective nature of CTA means that the contrast agent is injected into a peripheral systemic vein passing multiple capillary networks before reaching the portal venous system. Contrast detection will depend 
on the degree of contrast dilution, the sensitivity of the scanner's ability to detect the contrast and the timing of the acquisition of the scans relative to contrast injection. The pressure by which contrast agent is deposited in the liver will depend, to a large extent, on portal venous pressure. On the other hand, TFL-IOMP is highly likely to achieve far higher pressure during the hand injection as contrast is injected into an effectively closed system (with the shunt vessel ligated). This will lead to greater concentrations of contrast within the hepatic portal vasculature at higher pressure than that achievable by CTA, and will therefore increase the size and visibility of the intrahepatic portal veins, provided the shunt vessel has been adequately ligated. The choice of mesenteric vein tributary that was used for the technique did not appear to affect the identification of the intrahepatic portal vasculature, nor was there variation in the appearance of the intrahepatic vasculature based on shunt type with TFL-IOMP.

A greater number of dogs with shunts involving the right gastric vein were identified in this study (43 per cent) than in previous studies (for example, 21 per cent in a recent review (White, Shales \& Parry 2017). A definitive reason for this variation is not identified. However, it may be due 180 to the relatively small number of patients included in this study. This small sample size is a limitation of this study and, as a consequence, it is not possible to conclude definitely that these findings apply to all patients with congenital EHPPSs. For example, patients without intrahepatic portal vasculature (portal hypoplasia) were not available for inclusion in this study and it is, therefore, not possible to conclude whether findings from pre-operative CTA and TFL-IOMP would be significantly different in such cases.

The appearance of the intrahepatic vasculature varied between CTA and TFL-IOMP. CTA showed a reduction in intravascular contrast enhancement in cases where shunts involved the right gastric vein, whereas patients with this shunt type consistently had good intrahepatic 
vascular enhancement on TFL-IOMP. Variation in the pre-operative CTA appearance of the intrahepatic vasculature with shunts involving the right gastric vein has been described previously (Parry and White 2017). Briefly, preferential flow of contrast and streamlining of contrast agent within the portal vasculature may cause blood within the portal vein to mix incompletely, and remain streamlined in character, with discrete channels of flow permitting the liver to receive blood from discrete viscera. Whether the viscosity of the contrast agent plays a role in streamlining has, to the authors' knowledge, not been investigated. A laminar flow appearance of contrast on pre-ligation IOMP has been observed previously and this has been described as a potential source of variation in the appearance of the intrahepatic portal vasculature when using this imaging modality (Parry \& White 2017). Such a laminar flow appearance was not identified on any TFL-IOMP in this current study and its absence was considered likely due to supra-physiological pressures achieved by the hand injection of contrast agent into what was effectively a closed circuit. Further studies are required to investigate this phenomenon in more detail.

A further explanation as to why pre-operative CTA was outperformed by TFL-IOMP is given by the proportion of blood entering the liver from the portal vasculature in patients with a portosystemic shunt. In patients with a portosystemic shunt a proportion of the portal blood will bypass the liver entering directly into a systemic vein. In cases where the 'shunting' proportion of blood is high there will be a comparative reduction in intrahepatic portal blood flow. It is not surprising, therefore, that in patients with an EHPSS there would be a reduction in the documentation of the intrahepatic portal vasculature for CTA when compared to TFLIOMP.

Variation between the two modalities may also be influenced by a number of other factors. For 
example, the positioning of the patient at the time of the examination. For CTA examinations, patients were always positioned in sternal recumbency, and for TFL-IOMP, patients were invariably positioned in dorsal recumbency. Such differences in patient positioning are likely to be associated with differences in both intra-abdominal and intra-thoracic pressures, which in turn might have an effect on contrast enhancement of the portal system. Whether this alteration in patient position has any such effect is yet to be established. In addition, other factors might have effects on the differences in the contrast enhancement of the portal system between the two modalities; for example, an 'open' (TFL-IOMP) versus 'closed' (CTA) abdomen, and exteriorisation and the placement of traction on the small bowel and its mesentery when performing the IOMP. These factors were not specifically investigated in the current study, although standard, recognised techniques were used for both CTA and the TFLIOMP. A previous study comparing CTA with pre-manipulation and ligation of the shunt IOMP indicated no inherent compromise to contrast enhancement of the portal system between the two modalities (Parry \& White 2017). The same basic methodology for CTA and IOMP were utilised in the current study in an attempt to mitigate the potential issues associated with contrast enhancement of the portal system.

Further variation may be due to the timing of the study on CTA. In all cases, a bolus tracking procedure was undertaken, but even with strict adherence to protocol, some variation in visibility of portal vasculature due to variation in timing of triggering of the study is unavoidable. It is after all, a 'snap shot' of the portal vasculature, obtained in a transient time frame. This is not the case for TFL-IOMP, which is a dynamic study. Similarly, TFL-IOMP gives an instantaneous visualisation of the entire intrahepatic portal vasculature during the operation. CTA on the other hand produces a lot of data that takes time to analyse. Assessment of individual portal vein branches requires assessment of multiple stacked images, in native 
format, multiplanar reformatted (MPR) and maximum intensity projection (MIP).

Volume rendered images of the CTA images were not used in this study. This is because the smaller diameter of the intrahepatic vasculature and reduced contrast enhancement of the vessels (compared to the extrahepatic portal vasculature) meant that volume rendered images proved to be unsatisfactory for assessment.

TFL-IOMP was better at assessing the intrahepatic portal vasculature than both CTA and preoperative IOMP (as discussed in Parry \& White, 2017). Both White et al. (2003) and Lee et al. (2006) showed that intrahepatic portal vasculature is better documented after temporary shunt ligation, compared to pre-ligation, based on IOMP findings in dogs. Furthermore, Lee et al. (2006) confirmed that a well-developed intrahepatic portal vasculature identified on IOMP following the temporary full ligation of an EHPSS could be used as a positive prognostic indicator for clinical outcome. Lipscomb et al. (2009) showed similar findings in cats. Since CTA is a non-selective technique, contrast is not administered under pressure into the portal circulation as with IOMP and may be expected to underestimate the presence of portal vasculature (Zwingenberger et al. 2013). The findings of this current study appear to confirm these previous findings.

When using the visual assessment adopted in this paper, there was little difference between CTA and TFL-IOMP. The main differences were identified on a more subjective assessment between the two modalities. This is a limitation of the study. Other visual assessment scales were considered. A visual analogue scale was used by MacDonald et al. (2002). The study compared a visual analogue scale with a numeric scoring system. It was concluded that whilst both techniques were reproducible and repeatable, the numeric scoring scale possessed a 
number of inherent deficiencies that suggested it was not the method of choice for assessing IOMP. The visual analog scale was considered more accurate when assessing patients with either a very well developed or very poorly developed portal vasculature, although assessment was less reliable in those patients where the portal vasculature was of intermediate development. For these reasons, use of a visual analog scale was not considered appropriate in this study.

We conclude that in the majority of EHPSS shunt types pre-operative CTA identified intrahepatic portal arborisation to similar degree to that shown using TFL-IOMP. Subjectively, however, contrast enhancement and the size of the intrahepatic portal vasculature was considered best with TFL-IOMP. However, importantly, TFL-IOMP is undertaken at the time of shunt attenuation surgery and provides dynamic results which are instantaneously available at the time of surgery. Although not specifically investigated in the current study, as a consequence TFL-IOMP has a number of specific attributes not available with pre-operative CTA. These include the potential to measure portal mesenteric venous pressure, the confirmation that the shunting vessel has been correctly identified and ligated at the appropriate site and that only one shunting vessel is present (White et al. 2003, Lee et al. 2006, Lipscomb et al. 2009). These factors have been shown previously to be important in decision-making 278 and prognosis at the time of shunt attenuation surgery (White et al. 2003, Lee et al. 2006, Lipscomb et al. 2009). We, therefore, conclude that TFL-IOMP provides additional information to pre-operative CTA in the clinical management of patients with EHPSSs.

Conflict 282 of interest:

None of the authors of this article has a financial or personal relationship with other people or 
organizations that could inappropriately influence or bias the content of the paper.

\section{REFERENCES}

Lee, K. C., Lipscomb, V. J., Lamb, C. R., Gregory, S. P., Guitian, J. \& Brockman, D. J. (2006) Association of portovenographic findings with outcome in dogs receiving surgical treatment for single congenital portosystemic shunts: 45 cases (2000-2004). Journal of the American Veterinary Medical Association 229: 1122-1129

Lipscomb, V. J., Lee, K. C., Lamb, C. R. \& Brockman, D. J. (2009) Association of mesenteric portovenographic findings with outcome in cats receiving surgical treatment for single congenital portosystemic shunts. Journal of the American Veterinary Medical Association 234: $221-228$

Macdonald N. J., Burton, C. A. \& White, R. N. (2002) Comparison of visual analog and numeric scoring scales for assessing intraoperative mesenteric portovenography. Veterinary Radiology and Ultrasound 43: 534-540

Nelson, N. C. \& Nelson, L. L. (2011) Anatomy of extrahepatic portosystemic shunts in dogs as determined by computed tomography angiography. Veterinary Radiology and Ultrasonography 52: 498-506

Parry, A. T. \& White, R. N. (2015) Portal vein anatomy in the dog: comparison between computed tomographic angiography (CTA) and intraoperative mesenteric portovenography (IOMP). Journal of Small Animal Practice 56: 657-661 
Parry, A.T., and White, R.N., (2017) Comparison of computed tomographic angiography (CTA) and intraoperative mesenteric portovenography (IOMP) for extrahepatic portosystemic shunts. Journal of Small Animal Practice 58: 49-55

White, R. N., Macdonald, N. J. \& Burton, C. A. (2003) Use of intraoperative mesenteric portovenography in congenital portosystemic shunt surgery. Veterinary Radiology and Ultrasound 44: 514-521

White, R.N. \& Parry, A.T. (2013) Morphology of congenital portosystemic shunts emanating from the left gastric vein in dogs and cats. Journal of Small Animal Practice 54: 459-467

White, R.N. \& Parry, A.T. (2015) Morphology of congenital portosystemic shunts involving the right gastric vein in dogs. Journal of Small Animal Practice 55: 430-440

White, R.N. and Parry, A.T. (2016a) Morphology of splenocaval congenital portosystemic shunts in dogs and cats. Journal of Small Animal Practice 57: 28-32

White, R.N. \& Parry, A.T. (2016b) Morphology of congenital portosystemic shunts involving the left colic vein in dogs. Journal of Small Animal Practice 57: 247-254

White, R.N., Shales, C. \& Parry, A.T. (2017) New perspectives on the development of extrahepatic portosystemic shunts. Journal of Small Animal Practice doi: 10.1111/jsap.12728

Wolschrijn, C. F. (2010) The abdomen of the dog and cat. In: Textbook of veterinary anatomy. 
4th edn. Eds Dyce, K.M., Sack, W.O. and Wensing, C.J.G. Saunders Elsevier, St. Louis. p 450

Zwingenberger, A L. \& Schwarz, T. (2004) Dual-phase CT angiography of the normal canine portal and hepatic vasculature. Veterinary Radiology and Ultrasound 45: 117-124

Zwingenberger, A. L., Daniel, L., Steffey, M. A., Mayhew, P. D., Mayhew, K. N., Culp, W. T. N., Hunt, G. B., (2013) Correlation between liver volume, portal vascular anatomy, and hepatic perfusion in dogs with congenital portosystemic shunt before and after placement of ameroid constrictors. Veterinary Surgery 43: 926-934 